\title{
28 Research Square \\ Characterization of Pemigatinib as a Selective and Potent FGFR Inhibitor
}

Qianmeng Lin

Xiangya Hospital, Central South University

\section{Xiaojuan Chen}

Xiangya Hospital, Central South University

\section{Lingzhi Qu}

Xiangya Hospital, Central South University

\section{Ming Guo}

Xiangya Hospital, Central South University

\section{Hudie Wei}

Xiangya Hospital, Central South University https://orcid.org/0000-0003-2072-2169

\section{Shuyan Dai}

Xiangya Hospital, Central South University

\section{Longying Jiang}

Xiangya Hospital, Central South University https://orcid.org/0000-0002-3920-9878

Yongheng Chen ( $\nabla$ yonghenc@163.com )

Xiangya Hospital, Central South University https://orcid.org/0000-0001-8139-6892

\section{Article}

Keywords: pemigatinib, FGFR inhibitor, gatekeeper mutation, X-ray crystallography

Posted Date: February 16th, 2022

DOI: https://doi.org/10.21203/rs.3.rs-1279682/v1

License: (c) (1) This work is licensed under a Creative Commons Attribution 4.0 International License. Read Full License

Version of Record: A version of this preprint was published at Communications Chemistry on August 22nd, 2022. See the published version at https://doi.org/10.1038/s42004-022-00718-z. 


\section{Abstract}

FGFRs dysregulation is involved in a variety of tumorigenesis and development. Cholangiocarcinoma is closely related with FGFR aberrations, and pemigatinib is the first drug approved to target FGFR for the treatment of cholangiocarcinoma. Herein, we undertake biochemical and structural analysis on pemigatinib against FGFRs as well as gatekeeper mutations. The results show that pemigatinib is a potent and selective FGFR1-3 inhibitor. The extensive network of hydrogen bond and van der Waals contacts found in the FGFR1-pemigatinib binding mode accounts for the high potency. Pemigatinib is also potent against Val-to-lle gatekeeper mutation but cannot overcome Val-to-Met/Phe gatekeeper mutation in FGFR. Taken together, the inhibitory and structural profiles exemplified by pemigatinib may help to thwart Val-to-lle gatekeeper mutation-based resistance at earlier administration and to advance the further design and improvement for inhibitors toward FGFRs with gatekeeper mutation.

\section{Introduction}

Fibroblast growth factor receptors (FGFRs) belong to the tyrosine kinase receptor family ${ }^{1}$. It can regulate cell migration, proliferation, and cell differentiation by activating downstream signaling pathways, including the RAS-MAPK-ERK, PI3K-AKT and Janus kinase-signal transducer and activator of transcription (JAK-STAT) signaling pathways ${ }^{1-4}$. Genetic alterations in FGFRs would lead to the aberrations of the activated FGFR signaling pathway and further the establishment and development of a wide variety of cancers ${ }^{5-8}$. FGFR2 aberrations are found in $10-15 \%$ of patients with intrahepatic cholangiocarcinoma ${ }^{9}$. Approximately $50 \%$ of bladder cancers are associated with FGFR3 mutations, and $30 \%$ of hepatocellular carcinoma patients have abnormally increased FGFR4 expression ${ }^{10,11}$. Therefore, targeting the FGFR signaling pathway represents an attractive therapeutic target. Many small-molecule inhibitors are being discovered and approved by the FDA for patients harboring FGFR alterations, such as pemigatinib (Figure 1A), erdafitinib (Figure 1B) and infigratinib (Figure 1C).

Pemigatinib is the first targeted therapy approval by the FDA in April 2020 to treat patients with previously treated, unresectable, locally advanced, or metastatic cholangiocarcinoma and an FGFR2 fusion or other rearrangement ${ }^{12,13}$. In the Phase II FIGHT-202 trial (NCT02924376), pemigatinib yielded an objective response rate of $35.5 \%$ ( $2.8 \%$ complete responses) and a disease control rate of $82.0 \%{ }^{14}$. These encouraging outcomes underlie the potential benefit of pemigatinib in clinical use. It is supposed that pemigatinib would become a first-line treatment for these patients before mentioned. An ongoing clinical trial, FIGHT-302, will evaluate the efficacy and safety of pemigatinib therapy versus gemcitabine plus cisplatin combination therapy ${ }^{15}$. Additionally, pemigatinib is expected to treat other FGFR-driven malignancies, such as urothelial carcinoma, and relevant clinical trials are launched and undergoing in various countries worldwide ${ }^{12}$.

After an initial response to the therapies of tyrosine kinase inhibitors, acquired drug resistance gradually develops, correlated with therapy discontinuation and cancer advances, which can be mediated by the 
alternations in the protein-drug binding and/or the activation of bypass signaling ${ }^{16,17}$. Gatekeeper mutations in kinase domains represent a common theme, which maps at the beginning of the hinge region, curbs the accessibility of the inhibitor to the ATP pocket and contributes to the loss of effectiveness of the inhibitor, such as Abl T315I and EGFR T790M ${ }^{18-21}$. Ponatinib and Osimertinib, the third-generation tyrosine kinase inhibitors, are developed to overcome Abl T315I and EGFR T790M, respectively ${ }^{22,23}$. Therefore, overcoming resistance by gatekeeper mutations is a novel and subsequent direction of drug design and improvement for next-generation FGFR inhibitors. As the first-generation FGFR inhibitor, pemigatinib needs the further development so the deeper insight into the inhibitory and structural profiles of pemigatinib is necessary.

In this study, we aimed to explore the structural basis for the high potency of pemigatinib against FGFRs and predict the sensitivity to gatekeeper mutations. We undertook biochemical and structural analysis on pemigatinib against FGFRs and gatekeeper mutations. The results show that pemigatinib is capable of inhibiting FGFR1-3 and retains excellent potency against FGFR2 V564I but lower potency against FGFR4 and other gatekeeper mutations. Our results may provide some structural basis and design directions for further optimization of FGFR inhibitors.

\section{Results}

\section{The potent inhibition of Pemigatinib on FGFRs}

To confirm the inhibitory effect of pemigatinib on FGFR1-4, we performed kinase activity inhibition assays. As shown in Figure 1, pemigatinib exhibits potent inhibitions against FGFR1-3 with half maximal inhibitory concentrations $\left(\mathrm{IC}_{50}\right)$ of $0.2,1.2$ and $1.4 \mathrm{nM}$, respectively. Compared with FGFR1-3, the inhibition upon FGFR4 is greatly reduced ( 75 -fold less potent compared with FGFR1), with an $\mathrm{IC}_{50}$ of 15 nM. Meanwhile, we also carried out cellular proliferation assays in $\mathrm{Ba} / \mathrm{F} 3$ cells to further verify the inhibitory effect of pemigatinib. Pemigatinib had no impact on the growth of parental Ba/F3 cells with $\mathrm{IC}_{50}$ value exceeding $5 \mu \mathrm{M}$. It has great potency against the proliferation of FGFR1-3-translocated Ba/F3 cells ( $\left(\mathrm{IC}_{50}\right.$ of $1.2,0.3$ and $1.2 \mathrm{nM}$, respectively) but lower potency against the proliferation of FGFR4translocated $\mathrm{Ba} / \mathrm{F} 3$ cells $\left(\mathrm{IC}_{50}\right.$ of $\left.12 \mathrm{nM}\right)$, consistent with the results of kinase assays. Taken together, these results substantiate pemigatinib as a potent FGFR1-3 inhibitor.

\section{Structural Basis of Pemigatinib-FGFR1 Interaction}

To gain structural insights into the mechanism of FGFR inhibition by pemigatinib, the crystal structure of the pemigatinib/FGFR1 kinase domain was determined at a high resolution of $2.5 \AA$. The collection data in detail are shown in Table S1. The chemical structure of pemigatinib is described in Figure 1A, and its electron density and structural characteristics are well represented in the crystal structure with FGFR1 kinase domain (Figure 2A). Pemigatinib occupies the ATP-binding pocket of FGFR1, where the activation loop adopts a DFG-in conformation (Figure 2B), resembling other selective FGFR inhibitors. The pyrrolopyridine moiety forms two hydrogen bonds with the $\mathrm{N}-\mathrm{H}$ group and the carbonyl group of Ala564 in the hinge region of FGFR1 (Figure 2C). The difluoromethoxyphenyl ring is oriented perpendicular to the 
tricyclic scaffold and occupies the hydrophobic pocket containing the gatekeeper residue Val561. One of the methoxy oxygen atoms of the difluoromethoxyphenyl ring forms a hydrogen bond with the backbone nitrogen atom of Asp641 (Figure 2C). The morpholine solubilizing group extends away from the hinge region towards the solvent exposed region and does not make any specific interactions with FGFR1. Apart from direct interactions, pemigatinib binds to FGFR1 indirectly via the water molecule that links the oxygen atom of the tricyclic scaffold to the amino side chain of Lys514 and the carboxylate side chain of Asp641 (Figure S1). More interactions, including van der Waals forces, are also described in Figure S1. These results manifest that pemigatinib firmly binds FGFR1 via an extensive interaction network.

\section{Structural Comparison of Pemigatinib to Erdafitinib/Infigratinib in Complex with FGFR1}

To date, FGFR inhibitors approved by the FDA exclusively contain pemigatinib, erdafitinib and infigratinib. To help fully understand the profiles of these three drugs, we performed a structural comparison of their binding to the FGFR1 kinase domain. The activation loops of these three drugs binding FGFR1 are all kept in the DFG-in conformation (Figure 3A-C), indicating that they are classified as Type I inhibitors ${ }^{24,25}$. A 3,5-dimethoxylphenyl ring is observed in these three inhibitors that occupies the hydrophobic pocket via many van der Waals contacts. One of the methoxy oxygen atoms is involved in a hydrogen bond with Asp641, which increases the selectivity for FGFRs ${ }^{8}$. With regard to the molecular scaffold, the quinoxaline in erdafitinib formed one hydrogen bond with Ala564, whereas the tricyclic urea scaffold in pemigatinib and the N-aryl-N'-pyrimidin-4-yl urea scaffold in infigratinib formed two hydrogen bonds with Ala564 (Figure 3D-F). More hydrogen bonds formed could stabilize the complex conformations, which may explain why pemigatinib $\left(\mathrm{IC}_{50}\right.$ of $\left.0.2 \mathrm{nM}\right)$ and infigratinib $\left(\mathrm{IC}_{50}\right.$ of $\left.0.9 \mathrm{nM}\right)$ have more potency toward FGFRs than erdafitinib ( $\left(\mathrm{C}_{50}\right.$ of $\left.1.2 \mathrm{nM}\right){ }^{25,26}$. Moreover, they all possess a solubilizing group. As to pemigatinib, the solubilizing group is comprised of morpholine group, and to infigratinib and erdafitinib are ethyl piperazine and methyl pyrazole, respectively, which could increase their solubility and drug dissolution in the body ${ }^{8,25}$. These results show that the binding mode of pemigatinib in complex with FGFR1 is similar to that of erdafitinib/infigratinib.

\section{The Effect of FGFRs Gatekeeper Mutations on The Potency of Pemigatinib}

The question of whether gatekeeper mutations in the FGFR kinase domain have an influence on the inhibitory efficacy of pemigatinib has not been addressed. Thus, kinase activity inhibition assays were carried out to assess the sensitivity of pemigatinib to typical and frequent gatekeeper mutations, involving FGFR1 V561M, FGFR2 V564I/F and FGFR3 V555M (Figure 4). Unexpectedly, pemigatinib retains excellent potency against FGFR2 V564I with an $\mathrm{IC}_{50}$ of $7 \mathrm{nM}$ in the kinase assay, which exhibits merely a 4.7-fold reduction in inhibitory efficacy compared to the wild type (Figure 4A-B). Pemigatinib remains effective against FGFR2 V564l, possibly because the smaller isoleucine side chain causes less severe hindrance (Figure 4D). In contrast, the efficacy of pemigatinib is evidently diminished by the introduction of methionine to replace valine. It's found that pemigatinib exhibits $\sim 745$-fold lower potency against FGFR1 V561M (IC 50 of $149 \mathrm{nM}$ ), and 76-fold lower potency against FGFR3 V555M (IC 50 of $107 \mathrm{nM})$ (Figure 4A-B). Moreover, pemigatinib is vunerable to V564F mutation in FGFR2. A great reduction in 
efficacy for pemigatinib was observed in the V564F mutation, with an $\mathrm{IC}_{50}$ of $263 \mathrm{nM}$ ( 219-fold lower potency than FGFR1) (Figure 4A-B). Structural models suggest that pemigatinib-FGFR binding is more susceptible to disruption by the introduction of Met/Phe than lle, due to a more severe steric clash caused by the bulky Met/Phe (Figure 4E-F), indicating that less severe hindrance could preserve the inhibitory potency to overcome gatekeeper mutations. These results reveal that pemigatinib could remain inhibition against Val-to-Ile mutation but cannot overcome Val-to-Met/Phe mutation in FGFR.

\section{Discussion}

Pemigatinib is the first small molecule targeted drug to treat cholangiocarcinoma ${ }^{12}$. Despite slight loss of efficacy for FGFR4, pemigatinib has great potency against FGFR1-3, indicating that pemigatinib represents a potential therapy for other FGFR-driven malignancies. The related clinic trials have been undertaken to expand the application of pemigatinib ${ }^{12}$. Structural analysis shows that pemigatinib can be divided into three important chemical components according to their interactions with FGFR1. Firstly, the 3,5-dimethoxylphenyl ring that occupies the hydrophobic pocket is essential for the selectivity for FGFR, which may be enhanced by the introduction of fluorine/chlorine ${ }^{8}$. Secondly, the tricyclic urea scaffold features a hydrogen-bond interaction with the hinge region, which could serve as a template for further design and screening of drug structures of FGFR and/or other kinase receptor inhibitors. Thirdly, the solubilizing morpholine group regulates the lipophilicity and contributes to excellent rat and cynomolgus monkey PK profiles as well as abrogation of time-dependent inhibition issue ${ }^{8}$. This extensive network of pemigatinib-FGFR1 contact reinforces the strength of drug binding and accounts for the high potency of pemigatinib, providing an exemplification for the further design and optimization of FGFR inhibitors with high selectivity and high potency.

Further, the structural comparison of pemigatinib to erdafitinib and infigratinib in complex with FGFR1 highlights the importance of these three chemical components in drug design and optimization. 3,5dimethoxylphenyl ring, molecular scaffold and solubilizing group are all observed in these three drugs with extensive and indispensable interactions with FGFR1. Besides, they are classified as Type I inhibitors as their activation loops adopt a DFG-in conformation. These crystal structures provide a detail representation of common characteristics and nature of their interaction patterns for better understanding the functions of FGFR inhibitors.

Pemigatinib is the first-generation FGFR inhibitor. Based on previous experience with tyrosine kinase inhibitors ${ }^{27-30}$, resistance would occur due to the mutations within the kinase domain, such as Abl T315I and EGFR T790M ${ }^{18-21}$. In order to overcome these gatekeeper mutations, the third-generation kinase inhibitors have been developed, including ponatinib and Osimertinib ${ }^{22,23}$. Apart from Abl and EGFR, there are also some clinically-related gatekeeper mutations reported in FGFR, such as FGFR1 V561M, FGFR2 V564I/F, FGFR3 V555M and so on ${ }^{31}$. In our study, we find that pemigatinib can maintain activity against Val-to-lle gatekeeper mutation, however it cannot overcome Val-to-Met/Phe gatekeeper mutation in FGFR, which may attribute to the severe steric clash between the bulky Met/Phe and the 3,5-dimethoxylphenyl 
ring. Therefore, overcoming resistance may achieve by substitution of the 3,5-dimethoxylphenyl ring for a smaller group with larger space to accommodate the bulky gatekeeper residues.

In this study, we perform kinase activity inhibition assays and cellular proliferation assays to demonstrate that pemigatinib is a potent and selective FGFR1-3 inhibitor. The structural analysis provides the features and nature of the pemigatinib-FGFR1 binding pattern, accounting for the high potency of pemigatinib. Pemigatinib could sustain the potency against Val-to-lle gatekeeper mutation but cannot overcome Val-to-Met/Phe gatekeeper mutation, suggesting that the administration of pemigatinib at earlier stages of disease may evade Val-to-lle mutation-based resistance. Collectively, these structural and inhibitory profiles exemplified by pemigatinib may be applied to the exploration of the next-generation FGFR inhibitors to overcome gatekeeper mutation-based resistance.

\section{Methods}

\section{Plasmid Construction}

Human FGFRs were prepared as previously described ${ }^{32-35}$. Briefly, the kinase domains of FGFR1 (residues 458-765), FGFR2 (residues 453-770) and FGFR3 (residues 450-758) were respectively cloned into a modified pET28a expression vector with an N-terminal $6 \times$ His tag followed by a PreScission cleavage site. The mutant plasmids, FGFR1 C584S, FGFR1 V561M, FGFR2 V564I, FGFR2 V564F and FGFR3 V555M, were constructed by PCR using primers with the desired mutations.

\section{Protein expression and purification}

Plasmids were expressed in E. coli BL21 Rosetta cells. For FGFR1 C584S, Rosetta cells were co-expressed with YOPH to obtain non-phosphorylated proteins. The colony was inoculated into the liquid LB culture with $50 \mu \mathrm{g} / \mathrm{mL}$ kanamycin at $37^{\circ} \mathrm{C}$ and induced at $16^{\circ} \mathrm{C}$ for $18 \mathrm{~h}$ by the addition of $0.5 \mathrm{mM} \mathrm{IPTG}$ between $\mathrm{OD}_{600 \mathrm{~nm}}$ of $0.7 \sim 0.8$. The cells were harvested by 15 -min centrifugation at $3000 \mathrm{rpm}$. The pellets were resuspended in lysis buffer $(20 \mathrm{mM}$ Tris- $\mathrm{HCl}, \mathrm{pH} 8.0,500 \mathrm{mM} \mathrm{NaCl}, 20 \mathrm{mM}$ imidazole, $0.5 \mathrm{mM}$ TCEP$)$ and then lysed by a high-pressure homogenizer. The lysate supernatants were obtained after $35 \mathrm{~min}$ centrifugation at $18000 \mathrm{rpm}$ and incubated with equilibrated Ni-NTA beads (GE Healthcare) for $1.5 \mathrm{~h}$. Then, the beads were loaded into a gravity flow column and washed with lysis buffer containing $50 \mathrm{mM}$ imidazole. The target proteins were eluted with lysis buffer containing $250 \mathrm{mM}$ imidazole and digested with PreScission protease overnight to remove the N-terminal $6 \times$ His tag. Untagged FGFRs were further purified by anion exchange chromatography (GE Healthcare), and peak fractions collected were concentrated to $5-16 \mathrm{mg} / \mathrm{mL}$. For crystallization, the pooled FGFR1 C584S was put through a Superdex 200 column (GE Healthcare) in storage buffer (20 mM Tris-HCl, pH 8.0, $20 \mathrm{mM} \mathrm{NaCl}, 0.5 \mathrm{mM}$ TCEP).

\section{Kinase inhibition assay}

The kinase assays were performed by using the ADP-Glo (Promega) methodology. Proteins (0.025-0.2 $\mu \mathrm{M})$, pemigatinib (triple dilution method), ATP $(10 \mu \mathrm{M})$ and poly (4:1 Glu, Tyr) peptides (Abcam, UK) (10 $\mu \mathrm{M}$ ) were diluted with optimized kinase buffer (40 mM Tris- $\mathrm{HCl} \mathrm{pH} \mathrm{7.5,20} \mathrm{mM} \mathrm{MgCl} 2,20 \mathrm{mM} \mathrm{NaCl}, 0.1$ 
$\mathrm{mg} / \mathrm{mL}$ BSA, $1 \mathrm{mM}$ TCEP, and 4\% DMSO). The specific steps were prepared as previously described ${ }^{36}$. In short, the kinase reactions were initiated by mixing protein, inhibitor, ATP, and poly (4:1 Glu, Tyr) peptides, reacting at room temperature for 30 minutes, and then terminated by adding ADP-Glo after 40 min of incubation. Kinase activities were detected on a plate reader (Perkin Elmer) after the addition of detection reagent. $I_{50}$ values were calculated with log[Inhibitor] versus kinase activity ratio using GraphPad Prism software.

\section{Crystallization and structure determination}

The hanging drop vapor diffusion method was used for crystallization as previously described ${ }^{37,38}$. FGFR1/pemigatinib crystals were produced by micro-seeding FGFR1 crystal. The FGFR1 crystals were grown at $4{ }^{\circ} \mathrm{C}$ by mixing $0.8 \mu \mathrm{L}$ of protein solution with $0.8 \mu \mathrm{L}$ of crystallization buffer comprising $18 \%$ $(\mathrm{w} / \mathrm{v})$ PEG 8000, 0.2 M LiS04, and 0.1 M MES (pH 6.5). After one week, the crystals of FGFR1 were broken into microcrystals. FGFR1/pemigatinib crystals were produced similarly to the apo FGFR1 crystals but with the inhibitor molecule pre-incubated with FGFR1 in a 1:2 ratio overnight at $4{ }^{\circ} \mathrm{C}$ before micro-seeding and with identical crystallization conditions as apo FGFR1. The FGFR1/pemigatinib crystals were cryoprotected in the buffer supplemented with $20 \%$ glycerol and then flash-cooled in liquid nitrogen prior to data collection.

The X-ray diffraction data were collected in our lab, with HKL3000 employed for data integration and scaling ${ }^{39}$. The initial structures were solved by molecular replacement by Phaser from the PHENIX package with a search model from PDB entry $4 \mathrm{RWJ}{ }^{40}$. Further structure refinement and model building were performed by Phenix. refine and $\operatorname{Coot}^{39}$. Full details are described in the Table S1. PyMOL and LigPlot+ were used for structural descriptions and protein-ligand interaction representations, respectively ${ }^{41}$. The coordinates and structure factors have been deposited in the PDB under accession numbers 7 WCL.

\section{BaF3 Cell Lines Proliferation Assays}

Cell proliferation and viability were evaluated in parental Ba/F3 cells and TEL-FGFR1-4 Ba/F3 cell lines ${ }^{42}$. Cells were seeded in 96-cell plates at $2 \times 10^{3}$ cells/well and incubated with various concentrations of pemigatinib in a final volume of $100 \mu \mathrm{L}$ for $72 \mathrm{~h}$ at $37^{\circ} \mathrm{C}$. Subsequently, Cell Counting Kit-8 (Vazyme, China) was added, and after a 2-h incubation, the absorbance was measured at $450 \mathrm{~nm}$ using a multimode plate reader (Perkin Elmer). Each assay was performed in triplicate, and the $\mathrm{IC}_{50}$ values were calculated for the inhibitory potency of pemigatinib in vivo by GraphPad Prism 7.0.

\section{References}

1. Babina, I. S. \& Turner, N. C. Advances and challenges in targeting FGFR signalling in cancer. Nat Rev Cancer 17, 318-332, doi:10.1038/nrc.2017.8 (2017). 
2. Kimelman, D. \& Kirschner, M. Synergistic induction of mesoderm by FGF and TGF-beta and the identification of an mRNA coding for FGF in the early Xenopus embryo. Cel/ 51, 869-877, doi:10.1016/0092-8674(87)90110-3 (1987).

3. Corson, L. B., Yamanaka, Y., Lai, K. M. \& Rossant, J. Spatial and temporal patterns of ERK signaling during mouse embryogenesis. Development 130, 4527-4537, doi:10.1242/dev.00669 (2003).

4. Brewer, J. R., Mazot, P. \& Soriano, P. Genetic insights into the mechanisms of Fgf signaling. Genes Dev 30, 751-771, doi:10.1101/gad.277137.115 (2016).

5. Grose, R. \& Dickson, C. Fibroblast growth factor signaling in tumorigenesis. Cytokine Growth Factor Rev 16, 179-186, doi:10.1016/j.cytogfr.2005.01.003 (2005).

6. Beenken, A. \& Mohammadi, M. The FGF family: biology, pathophysiology and therapy. Nat Rev Drug Discov 8, 235-253, doi:10.1038/nrd2792 (2009).

7. Knights, V. \& Cook, S. J. De-regulated FGF receptors as therapeutic targets in cancer. Pharmacol Ther 125, 105-117, doi:10.1016/j.pharmthera.2009.10.001 (2010).

8. Wu, L. et al. Discovery of Pemigatinib: A Potent and Selective Fibroblast Growth Factor Receptor (FGFR) Inhibitor. J Med Chem 64, 10666-10679, doi:10.1021/acs.jmedchem.1c00713 (2021).

9. Arai, Y. et al. Fibroblast growth factor receptor 2 tyrosine kinase fusions define a unique molecular subtype of cholangiocarcinoma. Hepatology 59, 1427-1434, doi:10.1002/hep.26890 (2014).

10. Ho, H. K. et al. Fibroblast growth factor receptor 4 regulates proliferation, anti-apoptosis and alphafetoprotein secretion during hepatocellular carcinoma progression and represents a potential target for therapeutic intervention. J Hepato/ 50, 118-127, doi:10.1016/j.jhep.2008.08.015 (2009).

11. Cappellen, D. et al. Frequent activating mutations of FGFR3 in human bladder and cervix carcinomas. Nat Genet 23, 18-20, doi:10.1038/12615 (1999).

12. Hoy, S. M. Pemigatinib: First Approval. Drugs 80, 923-929, doi:10.1007/s40265-020-01330-y (2020).

13. Weng, Q., Tan, W., Yu, R. Y., Xu, R. A. \& Chen, Y. A novel bioanalytical method for the quantification of pemigatinib in rat plasma by UPLC-MS/MS. J Pharm Biomed Anal 202, 114137, doi:10.1016/j.jpba.2021.114137 (2021).

14. Abou-Alfa, G. K. et al. Pemigatinib for previously treated, locally advanced or metastatic cholangiocarcinoma: a multicentre, open-label, phase 2 study. Lancet Onco/ 21, 671-684, doi:10.1016/S1470-2045(20)30109-1 (2020).

15. Bekaii-Saab, T. S. et al. FIGHT-302: first-line pemigatinib vs gemcitabine plus cisplatin for advanced cholangiocarcinoma with FGFR2 rearrangements. Future Oncol 16, 2385-2399, doi:10.2217/fon2020-0429 (2020).

16. Krook, M. A. et al. Fibroblast growth factor receptors in cancer: genetic alterations, diagnostics, therapeutic targets and mechanisms of resistance. Br J Cancer 124, 880-892, doi:10.1038/s41416020-01157-0 (2021).

17. Schram, A. M., Chang, M. T., Jonsson, P. \& Drilon, A. Fusions in solid tumours: diagnostic strategies, targeted therapy, and acquired resistance. Nat Rev Clin Oncol 14, 735-748, 
doi:10.1038/nrclinonc.2017.127 (2017).

18. Liu, Y., Shah, K., Yang, F., Witucki, L. \& Shokat, K. M. A molecular gate which controls unnatural ATP analogue recognition by the tyrosine kinase v-Src. Bioorg Med Chem 6, 1219-1226, doi:10.1016/s0968-0896(98)00099-6 (1998).

19. O'Hare, T. et al. AP24534, a pan-BCR-ABL inhibitor for chronic myeloid leukemia, potently inhibits the T315I mutant and overcomes mutation-based resistance. Cancer Cell 16, 401-412, doi:10.1016/j.ccr.2009.09.028 (2009).

20. Andrews Wright, N. M. \& Goss, G. D. Third-generation epidermal growth factor receptor tyrosine kinase inhibitors for the treatment of non-small cell lung cancer. Transl Lung Cancer Res 8, S247S264, doi:10.21037/tlcr.2019.06.01 (2019).

21. Shah, N. P. et al. Overriding imatinib resistance with a novel ABL kinase inhibitor. Science 305, 399401, doi:10.1126/science.1099480 (2004).

22. Soo, R. A. et al. A randomised phase II study of osimertinib and bevacizumab versus osimertinib alone as second-line targeted treatment in advanced NSCLC with confirmed EGFR and acquired T790M mutations: the European Thoracic Oncology Platform (ETOP 10-16) BOOSTER trial. Ann Oncol, doi:10.1016/j.annonc.2021.11.010 (2021).

23. Parker, W. T. et al. The impact of multiple low-level BCR-ABL1 mutations on response to ponatinib. Blood 127, 1870-1880, doi:10.1182/blood-2015-09-666214 (2016).

24. Patani, H. et al. Landscape of activating cancer mutations in FGFR kinases and their differential responses to inhibitors in clinical use. Oncotarget 7, 24252-24268, doi:10.18632/oncotarget.8132 (2016).

25. Guagnano, V. et al. Discovery of 3-(2,6-dichloro-3,5-dimethoxy-phenyl)-1-\{6-[4-(4-ethyl-piperazin-1-yl)phenylamin o]-pyrimidin-4-yl\}-1-methyl-urea (NVP-BGJ398), a potent and selective inhibitor of the fibroblast growth factor receptor family of receptor tyrosine kinase. J Med Chem 54, 7066-7083, doi:10.1021/jm2006222 (2011).

26. Perera, T. P. S. et al. Discovery and Pharmacological Characterization of JNJ-42756493 (Erdafitinib), a Functionally Selective Small-Molecule FGFR Family Inhibitor. Mol Cancer Ther 16, 1010-1020, doi:10.1158/1535-7163.MCT-16-0589 (2017).

27. Hochhaus, A. et al. Roots of clinical resistance to STI-571 cancer therapy. Science 293, 2163 (2001).

28. Choi, Y. L. et al. EML4-ALK mutations in lung cancer that confer resistance to ALK inhibitors. $N$ Engl J Med 363, 1734-1739, doi:10.1056/NEJMoa1007478 (2010).

29. Kobayashi, S. et al. EGFR mutation and resistance of non-small-cell lung cancer to gefitinib. $N$ Engl J Med 352, 786-792, doi:10.1056/NEJMoa044238 (2005).

30. Yue, S. et al. FGFR-TKI resistance in cancer: current status and perspectives. J Hematol Oncol 14, 23, doi:10.1186/s13045-021-01040-2 (2021).

31. Nakanishi, Y. et al. The fibroblast growth factor receptor genetic status as a potential predictor of the sensitivity to CH5183284/Debio 1347, a novel selective FGFR inhibitor. Mol Cancer Ther 13, 25472558, doi:10.1158/1535-7163.MCT-14-0248 (2014). 
32. Wu, D. et al. LY2874455 potently inhibits FGFR gatekeeper mutants and overcomes mutation-based resistance. Chemical communications (Cambridge, England) 54, 12089-12092, doi:10.1039/c8cc07546h (2018).

33. Wlodawer, A. et al. Crystal Structure of the FGFR4/LY2874455 Complex Reveals Insights into the Pan-FGFR Selectivity of LY2874455. PLoS One 11, doi:10.1371/journal.pone.0162491 (2016).

34. Ni, F. et al. Remarkably Stereospecific Utilization of ATP a,ß-Halomethylene Analogues by Protein Kinases. J. Am. Chem. Soc. 139, 7701-7704, doi:10.1021/jacs.7b03266 (2017).

35. Rezende Miranda, R. et al. Development of a Potent and Specific FGFR4 Inhibitor for the Treatment of Hepatocellular Carcinoma. J. Med. Chem. 63, 11484-11497, doi:10.1021/acs.jmedchem.0c00044 (2020).

36. Zhou, Z. et al. Characterization of FGF401 as a reversible covalent inhibitor of fibroblast growth factor receptor 4. Chem. Commun. 55, 5890-5893, doi:10.1039/c9cc02052g (2019).

37. Guo, M. et al. Characterization of ibrutinib as a non-covalent inhibitor of SRC-family kinases. Bioorg. Med. Chem. Lett. 34, doi:10.1016/j.bmcl.2020.127757 (2021).

38. Deng, W. et al. Investigation of Covalent Warheads in the Design of 2-Aminopyrimidine-based FGFR4 Inhibitors. ACS Med. Chem. Lett. 12, 647-652, doi:10.1021/acsmedchemlett.1c00052 (2021).

39. Adams, P. D. et al. The Phenix software for automated determination of macromolecular structures. Methods 55, 94-106, doi:10.1016/j.ymeth.2011.07.005 (2011).

40. Sohl, C. D., Ryan, M. R., Luo, B., Frey, K. M. \& Anderson, K. S. Illuminating the molecular mechanisms of tyrosine kinase inhibitor resistance for the FGFR1 gatekeeper mutation: the Achilles' heel of targeted therapy. ACS Chem Bio/ 10, 1319-1329, doi:10.1021/acschembio.5b00014 (2015).

41. Laskowski, R. A. \& Swindells, M. B. LigPlot+: multiple ligand-protein interaction diagrams for drug discovery. Journal of chemical information and modeling 51, 2778-2786, doi:10.1021/ci200227u (2011).

42. Duensing, A. et al. Targeting Wild-Type and Mutationally Activated FGFR4 in Rhabdomyosarcoma with the Inhibitor Ponatinib (AP24534). PLoS One 8, doi:10.1371/journal.pone.0076551 (2013).

\section{Figures}


A<smiles>CCN1C(=O)N(c2c(F)c(OC)cc(OC)c2F)Cc2cnc3[nH]c(CN4CCOCC4)cc3c21</smiles>

Pemigatinib (INCB054828)

D

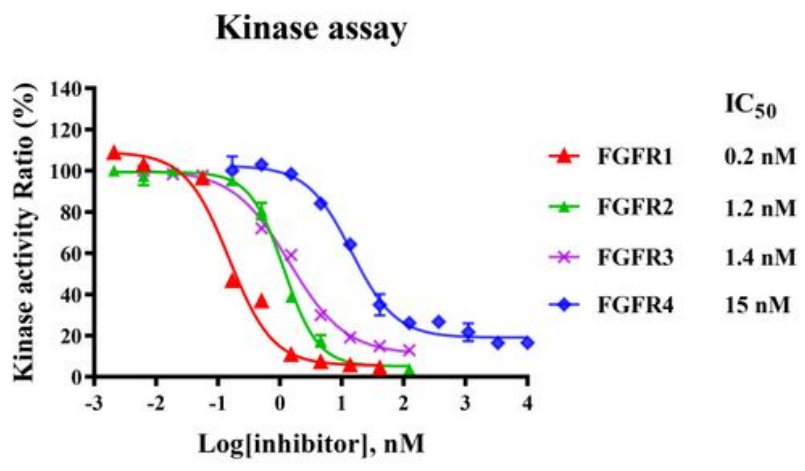

B<smiles>CCN1CCN(c2ccc(Nc3cc(N(C)C(=O)Nc4c(Cl)c(OC)cc(OC)c4Cl)ncn3)cc2)CC1</smiles>

Erdafitinib (JNJ-42756493)
C

E
Infigratinib (BGJ398)
$\mathrm{Ba} / \mathbf{F} 3$ cells

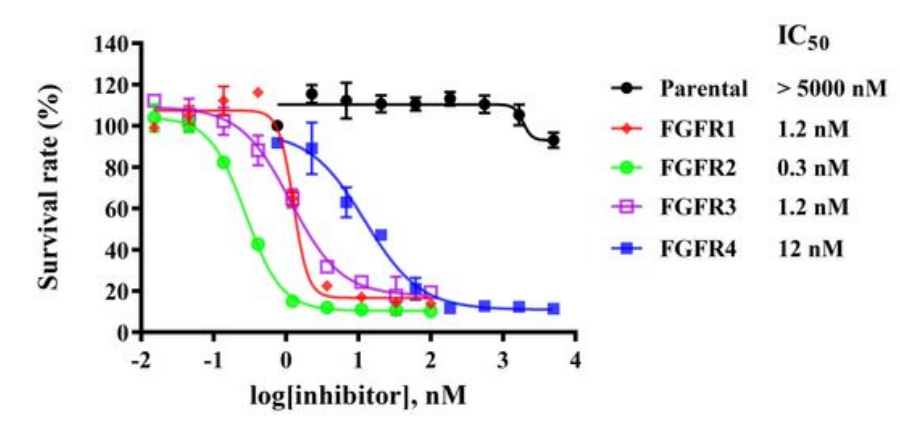

Figure 1

Legend not included with this version. 


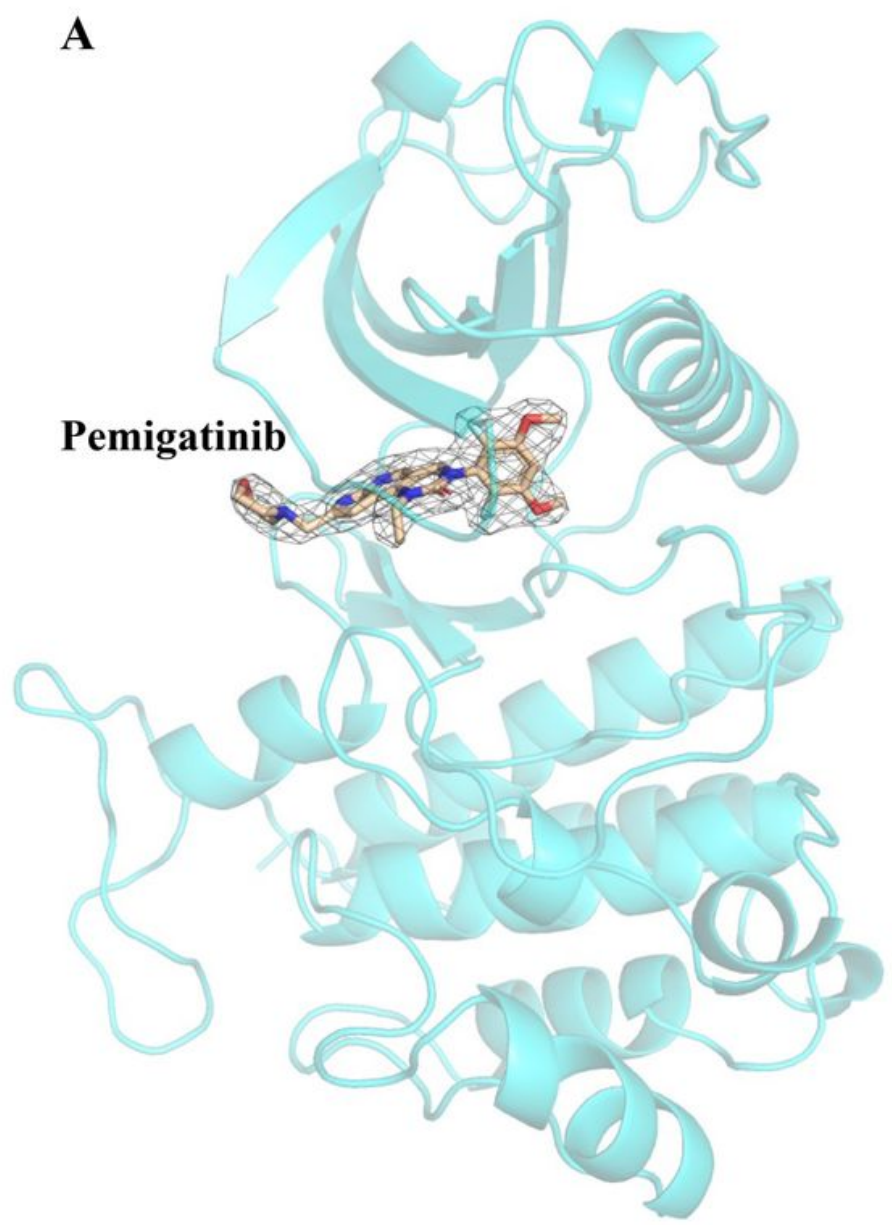

B

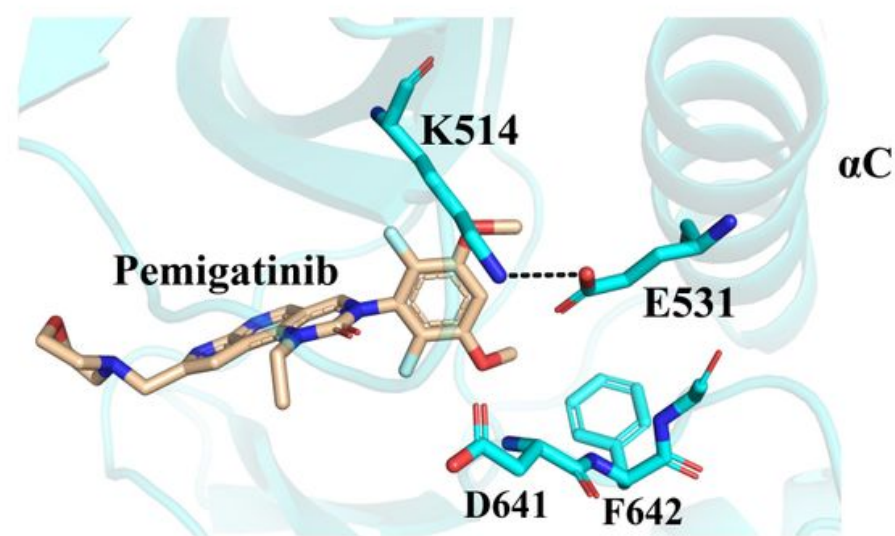

C

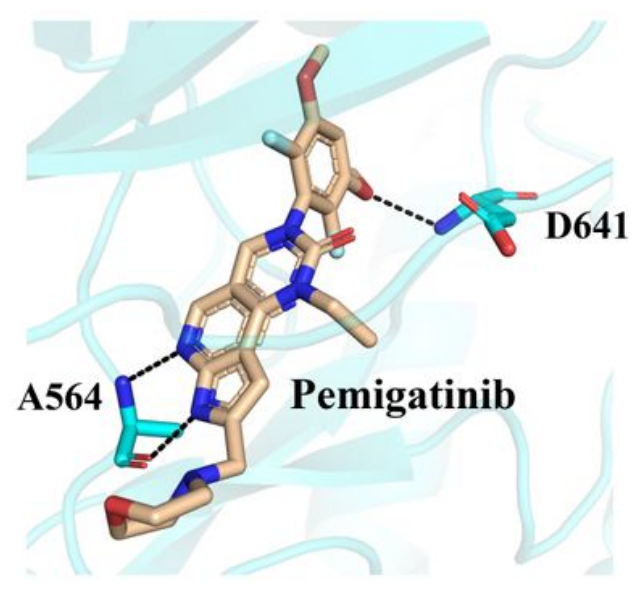

Figure 2

Legend not included with this version. 
A

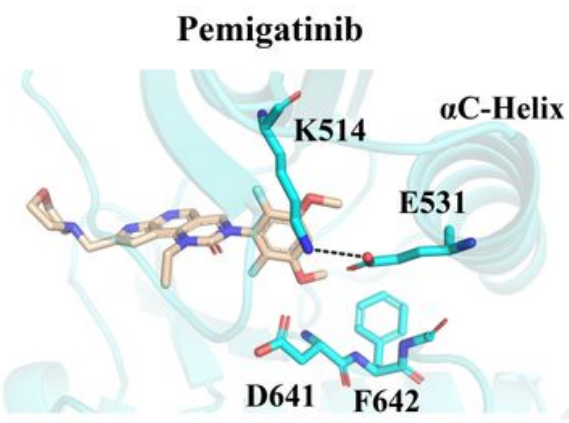

D

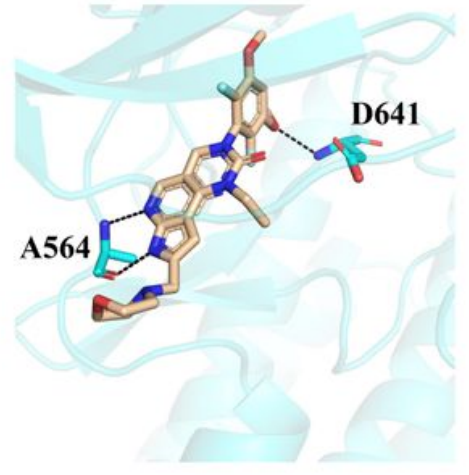

B

Erdafitinib

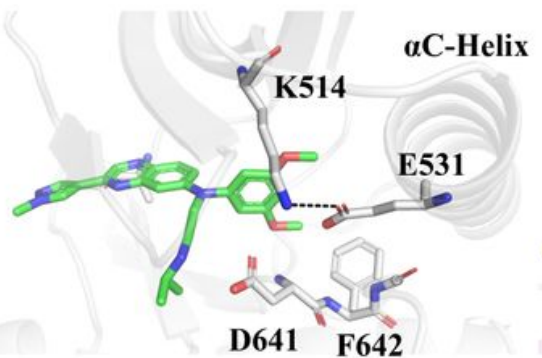

E

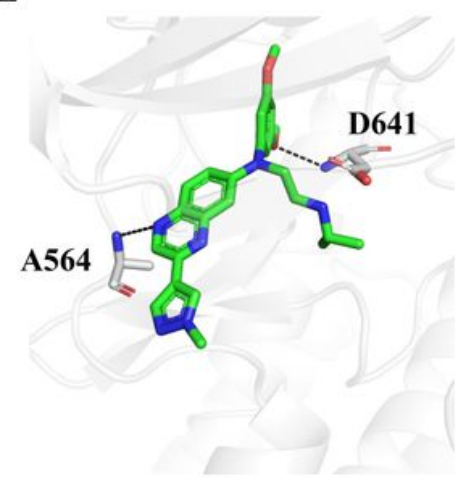

C Infigratinib

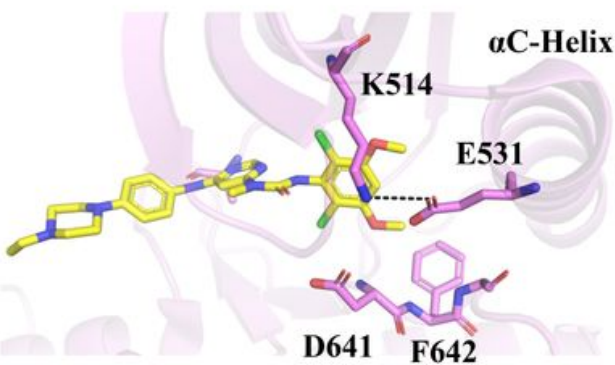

F

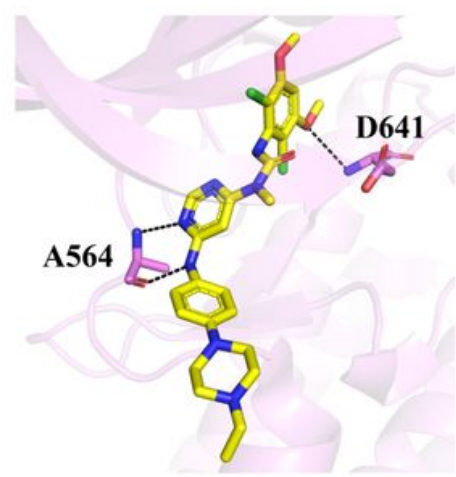

\section{Figure 3}

Legend not included with this version.

A

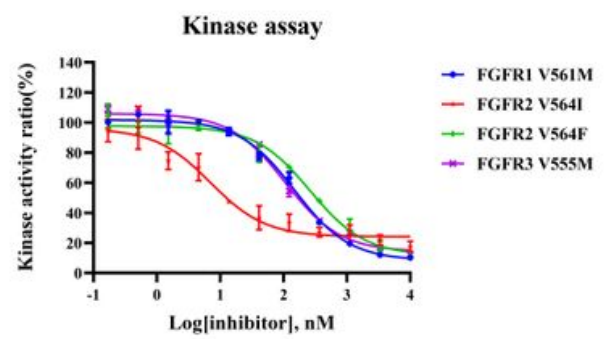

B

\begin{tabular}{lcc}
\hline \multirow{2}{*}{ Mutants } & \multicolumn{2}{c}{ Kinase inhibitory activity ( $\left.\mathrm{IC}_{50}\right)$} \\
\cline { 2 - 3 } & Pemigatinib $(\mathrm{nM})$ & mutant/wild-type \\
\hline FGFR1 V561M & 149 & 745 \\
FGFR2 V564I & 7 & 4.7 \\
FGFR2 V564F & 263 & 219 \\
FGFR3 V555M & 107 & 76 \\
\hline
\end{tabular}

C

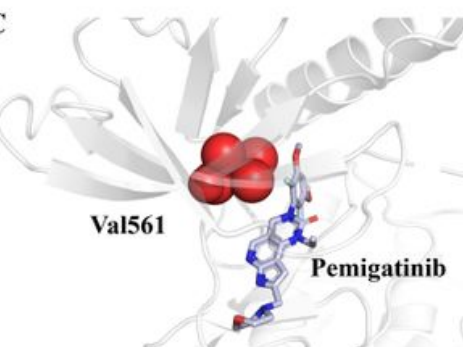

E

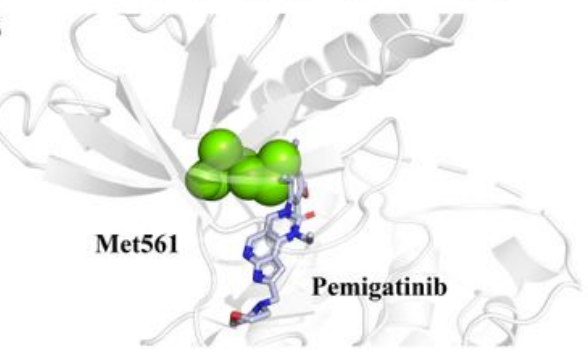

D

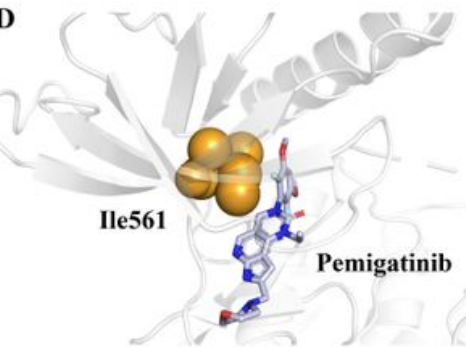

F

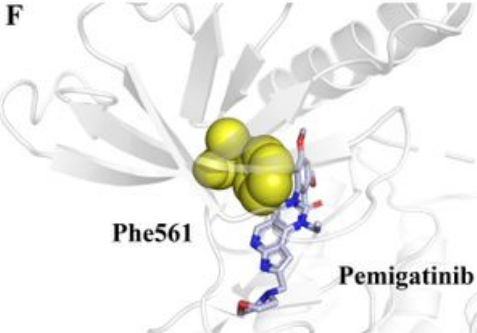

Figure 4

Legend not included with this version.

\section{Supplementary Files}


This is a list of supplementary files associated with this preprint. Click to download.

- FGFR1pemigatinibrefine14coot2.txt

- D1300026464valreportfullannotateP1.pdf

- FigureS1.pdf

- Tables1.docx 